\title{
Comparative evaluation between two nutritional supplements in the improvement of telogen effluvium
}

This article was published in the following Dove Press journal:

Clinical, Cosmetic and Investigational Dermatology

\author{
Flávia Alvim Sant'Anna \\ Addor ${ }^{\prime}$ \\ Ludmila Coelho Donato \\ Camila Sirieiro Abreu Melo \\ 'MEDCIN Research, Osasco, Brazil; \\ ${ }^{2}$ Clinical Research, Farmoquímica S.A, \\ Rio de Janeiro, Brazil
}

Correspondence: Flavia Alvim Sant'Anna Addor

178th Attilio Delanina St.

Osasco, São Paulo, Brasil 6023-010

Email flavia.addor@medcin.com.br
Purpose: Telogen effluvium (TE) is defined as a diffuse hair loss characterized by shortening of the anagen phase and precipitation of the telogen phase, with a consequent reduction of total hair volume. Nutritional supplementation is indicated under conditions in which TE is related to dietary disorders. The aim of this clinical study was to compare the efficacy of two different supplements in monotherapy for TE.

Patients and methods: Female adult patients were randomized to receive two oral nutritional supplements (group 1: a supplement composed of zinc, biotin, iron, vitamins A, C, E, and B complex, folic acid, magnesium, and amino acids of keratin and collagen and group 2: calcium pantothenate cystine, thiamine nitrate, medicinal yeast, keratin, and aminobenzoic acid) to treat hair loss for 180 days. They were evaluated clinically and by digital trichoscopy.

Results: Clinical evaluation showed significant clinical improvement $(P<0.05)$ for the evaluated parameters: hair loss, hair volume, density of hair (scalp cover), hair shine, hair strength, in 180 days. At 90 days evaluation, group 1 showed significant improvement for all parameters, while group 2 did not show any significant improvement for hair shine and hair strength. In the digital trichoscopy, there was a significant improvement only in group $1(11.09 \% \times 7.76 \%)$ after 180 days. Conclusion: In idiopathic TE, the nutritional component should be suspected; the supplementation of an association of nutrients in recommended daily intake can lead to significant improvement of the condition from the first trimester of use. The use of an association with proven efficacy and a safety profile and posologic convenience facilitate its indication and patient adherence.

Keywords: telogen effluvium, supplementation, trichoscopy, hair loss

\section{Introduction}

Telogen effluvium (TE) is defined as diffuse hair loss characterized by shortening of the anagen phase and precipitation of the telogen phase, with consequent reduction of the total hair volume. ${ }^{1}$

Its importance is related to its high occurrence in females, being the most common cause of alopecia in this group of patients. ${ }^{2}$

This change in hair cycle is related to various causes, and its severity is related to the time of exposure and severity of the causal factor. In addition, individual predisposition may also be an aggravating factor. ${ }^{3}$

Nutritional factor is one among the most described causes of this condition. In addition to proper nutritional diseases with hair repercussions, such as iron deficiency anemia or enteropathic acrodermatitis, malnutrition itself can lead to TE. ${ }^{4}$

Nutritional supplementation is indicated under conditions in which TE is related to dietary disorders, such as malabsorption, diets for weight loss, etc. Other etiological 
factors, such as childbirth, systemic diseases, can occur with deficits of certain nutrients, leading to worsening alopecia. ${ }^{5}$

As compounds with nutrients are always used as coadjuvants, their true role is difficult to prove. Monotherapy studies are scarce in the literature, especially comparative ones. ${ }^{6-8}$

Eximia Fortalize Kera $\mathrm{D}^{\circledR}$ (vitamins A, C, E, D, B [B1, B2, B3, B5, B6, B7 biotin, folic acid, and B12], zinc, iron, magnesium, and essential and nonessential amino acids) is a nutritional supplement with involved in keratin and collagen synthesis (Farmoquimica S.A, Rio de Janeiro, Brazil); these nutrients are somehow important in keratinogenesis and hair cycle maintenance, including dermal papillae integrity.

Pantogar $^{\circledR}$ (Keratin, Cystine, Paraminobenzoic acid, Medical yeast, Thiamine nitrate, Calcium pantothenate) is one of the most traditional and studied supplements indicated in hair loss, composed of some nutrients and chemical ingredients, being indicated for hair growth, hair and nail resistance, and hair loss.

In the present study, these two formulations available in the Brazilian market with different nutrient associations were comparatively evaluated in patients with idiopathic TE in a blind, randomized clinical trial.

\section{Purpose}

The purpose of this study was to investigate the effect of a nutritional supplement (Eximia Fortalize Kera $D^{\circledR}$ ) on the improvement of signs and symptoms of TE in relation to an oral drug used for the same purpose, through clinical evaluation and by digital trichoscopy (FotoFinder Dermoscope; FotoFinder Systems GmbH, Bad Birnbach, Germany), to evaluate hair density.

\section{Ethical aspects}

The present study was approved by an independent ethics committee of the São Francisco University (em 08/28/2015, CAAE: 48395415.4.0000.5514, advice 1.204.879; http:// aplicacao.saude.gov.br/plataformabrasil/login.jsf). All volunteers received detailed explanation of the study and provided written informed consent for participation in the study.

\section{Patients and methods}

The study protocol was initiated on September 26, 2016, and ended on September 4, 2017, at a private clinical research laboratory (Medcin Pesquisa, São Paulo, Brazil).

This was a prospective, comparative, randomized, blind study. A total of 199 female participants were screened; 120 participants aged between 18 and 60 years with TE for $\geq 6$ months were further selected, and clinically and instrumen- tally evaluated by a digital phototrichogram, with a proportion of telogen hairs over $20 \%$, and digital trichoscopy, discarding other causes of alopecia.

Besides the diagnostic procedures, all the patients agreed to avoid performing hair procedures throughout the study; they also had normal levels of androgenic and thyroid hormones; iron deficiency anemia was excluded. Blood count, ferritin, dehydroepiandrosterone sulfate, dihydrotestosterone, free testosterone, thyroxine, and thyroid-stimulating hormone tests were performed in all patients. Noninclusion criteria were androgenetic alopecia, alopecia areata, pregnancy risk, lactation, other alopecias, use of hormones, nonhormonal anti-inflammatory drugs, and corticosteroids for up to 4 weeks before inclusion, or immunosuppressants 3 months before inclusion, use of androgenic contraceptives, active systemic or cutaneous pathologies, as well as hair esthetic treatments and procedures for up to 4 weeks before the study.

All the patients included were evaluated clinically with a 4-point scale (where 0 represented the absence of an indication and 3 referred to an intense and the worst indication) about hair loss, hair volume (hair diameter), density of hair (scalp cover), and hair quality, whose parameters were flexibility, tensile strength, tip breakage, hair shine, and hair strength. They also answered a 4-point scale questionnaire (where 0 denoted the absence of an indication and 3 referred to an intense and the worst sign) about hair loss, hair shine, and hair strength.

Once included, the patients were randomized in two treatment groups:

- Group 1:60 participants used the tested product (Eximia Fortalize Kera $\mathrm{D}^{\circledR}$ ), composed of zinc, biotin, iron, vitamins A, C, E, and B complex, folic acid, magnesium, and amino acids of keratin and collagen;

- Group 2: 60 participants used the comparator product, composed of calcium pantothenate cystine, thiamine nitrate, medicinal yeast, keratin, and aminobenzoic acid $\left(\right.$ Pantogar $\left.^{\circledR}\right)$.

After data collection, the product was dispensed, and patients in group 1 were instructed to take one tablet a day for 6 months, and patients in group 2, three tablets a day during the same period, according to the package instructions.

Participants returned after 45, 135, and 180 days for adherence evaluation accountability used and usage diary information; in 90, 135 \pm 2 , and 180 for clinical, subjective, and instrumental evaluation. All return dates had a tolerance of \pm 2 days provided in the protocol.

Clinical evaluation investigated the following items, using a 4-point scale for intense improvement, mild improvement, 
no effect, or worsening: hair loss, hair volume (hair diameter), density of hair (scalp cover), hair quality, whose evaluated parameters were flexibility, tensile strength, tip breakage, hair shine, and hair strength.

Subjective evaluation included the following items, using a 4-point scale for intense improvement, mild improvement, no effect, or worsening: hair fall, hair shine, hair strength, comfort of use in terms of the amount of tablets/capsules ingested per day, ease of swallowing of tablets/capsules, residual taste of tablets/capsules.

At all visits, the safety of treatment use was assessed, and an investigation was conducted on possible adverse reactions; all adverse events had to be reported immediately to the research center.

Student's $t$-test for matched data was applied to identify whether the differences between means/medians from experimental times to continuous variables were statistically significant. To compare the means/medians of the test product with the comparator of these variables, Student's $t$-test was used. Comparisons of the experimental times for ordinal variables were performed using the Wilcoxon test for matched data and the Mann-Whitney $U$ test was used to compare the medians of the test product with the comparator product. The CI considered was $95 \%$.

\section{Results}

Ninety-two participants completed the study with valid data. Some participants did not complete the study due to factors that were not related to the treatments, reported as loss of interest/adherence due to the duration of the study, unavailability to attend visits, and unrelated personal reasons. Groups
1 and 2 had 47 and 49 participants, respectively, who were able to complete the study. No significant differences were detected between the products for probable or proven and total adverse events. In group 1, one patient referred mild nausea; in group 2, two patients referred mild gastrointestinal signs (dyspepsia and epigastralgia); no treatment interruption was needed.

\section{Treatment adherence}

Both groups adhered to the treatment, with no difference between them at the end of the treatment $(P=0.440)$.

\section{Clinical evaluation: 90 days}

Group 1 showed statistically significant improvements $(P<0.05)$ for all items evaluated; group 2, treated with the comparator, presented significant improvements for all items except hair shine and hair strength, as observed in Table 1 .

\section{Clinical evaluation: I 80 days}

Both groups presented a statistically significant improvement for all items evaluated, according to Table 2.

\section{Comparative evaluation between groups at 90 and 180 days}

There was no statistically significant difference $(P>0.05)$ between treatments in none of the evaluated items after 90 days. After 180 days, group 1 presented statistically significant superior results $(P<0.05)$ for the clinical parameters of hair quality, hair shine, and hair strength compared to group 2 (comparator product).

Table I Percentage of participants with some improvement under items evaluated in both groups after 90 days ( $\mathrm{n}=92$ )

\begin{tabular}{|c|c|c|c|c|c|c|c|c|c|c|c|c|}
\hline \multirow[t]{2}{*}{ D90 } & \multicolumn{2}{|c|}{ Hair loss } & \multicolumn{2}{|c|}{ Hair volume } & \multicolumn{2}{|c|}{ Density of hair } & \multicolumn{2}{|c|}{ Quality of hair } & \multicolumn{2}{|c|}{ Hair shine } & \multicolumn{2}{|c|}{ Hair strength } \\
\hline & $\mathbf{I}$ & II & $\mathbf{I}$ & II & I & II & $\mathbf{I}$ & II & I & II & $\mathbf{I}$ & II \\
\hline Clinical improvement (\%) & 76.6 & 60.0 & 51.0 & 42.2 & 48.9 & 33.3 & 53.1 & 40.0 & 36.1 & 22.2 & 40.4 & 31.1 \\
\hline$P$-value & $<\left.0.00\right|^{a}$ & $<0.00 I^{\mathrm{a}}$ & $<\left.0.00\right|^{a}$ & $0.003^{\mathrm{a}}$ & $<\left.0.00\right|^{a}$ & $0.014^{\mathrm{a}}$ & $<0.00 I^{a}$ & $0.016^{\mathrm{a}}$ & $0.042^{\mathrm{a}}$ & 0.856 & $0.008^{\mathrm{a}}$ & 0.076 \\
\hline
\end{tabular}

Notes: a Level of significance of $5 \%$. Hypothesis: non-inferiority between treatments.

Abbreviations: I, group treated with Eximia Fortalize Kera $\mathrm{D}^{\circledR}$; II, group treated with comparator; D90, 90-day evaluation.

Table 2 Percentage of participants with some improvement under items evaluated in both groups after 180 days ( $\mathrm{n}=92)$

\begin{tabular}{|c|c|c|c|c|c|c|c|c|c|c|c|c|}
\hline \multirow[t]{2}{*}{ DI80 } & \multicolumn{2}{|c|}{ Hair loss } & \multicolumn{2}{|c|}{ Hair volume } & \multicolumn{2}{|c|}{ Hair density } & \multicolumn{2}{|c|}{ Quality of hair } & \multicolumn{2}{|c|}{ Hair shine } & \multicolumn{2}{|c|}{ Hair strength } \\
\hline & $\mathbf{I}$ & II & I & II & $\mathbf{I}$ & II & $\mathbf{I}$ & II & $\mathbf{I}$ & II & $\mathbf{I}$ & II \\
\hline Clinical improvement (\%) & 87.2 & 82.2 & 59.5 & 53.3 & 59.5 & 55.5 & 72.3 & 51.1 & 59.5 & 40.0 & 70.2 & 53.3 \\
\hline$P$-value & $<0.001^{a}$ & $<0.00 I^{a}$ & $<0.001^{a}$ & $<0.001^{a}$ & $<0.001^{a}$ & $<0.00 \mathrm{I}^{\mathrm{a}}$ & $<0.00 I^{a}$ & $<0.001^{a}$ & $<0.001^{a}$ & $0.005^{\mathrm{a}}$ & $<0.001^{a}$ & $<0.001^{a}$ \\
\hline
\end{tabular}

Notes: aLevel of significance of $5 \%$. Hypothesis: non-inferiority between treatments.

Abbreviations: I, group treated with Eximia Fortalize Kera $\mathrm{D}^{\circledR}$; II, group treated with comparator; DI80, 180-day evaluation. 


\section{Evaluation by digital trichoscopy}

The instrumental parameter used was hair density, calculated automatically by the Trichoscale (FotoFinder Systems, Inc., Germany) software after scraping and counting the hair, the results of which are summarized in Table 3.

According to the results obtained in the digital trichoscopy evaluation, there was no significant difference in the increase in hair density after 90 days; however, in the 180-day evaluation, there was a significant increase in the mean hair density for group 1 , of $11.9 \%$.

\section{Subjective evaluation}

Both treatments were positively evaluated by the study participants, in the comparison between groups, after 90 days (D90) of use.

There was, however, a statistically significant superiority $(P<0.05)$ for group 1 in the evaluations for the parameters: hair strength, residual taste, and convenience of use in terms of the amount of tablets/capsules ingested per day after 90 days of use, according to Table 4.
At the end of the study, in the 180-day evaluation (Table 5), the results are similar, except for the convenience of usage parameter in the amount of tablets/capsules ingested per day, where the treatment in group 1 again demonstrated superiority over group 2, with the comparator product, in a statistically significant fashion.

\section{Discussion}

TE is one of the most frequent causes of alopecia in medical practice, although its prevalence is underestimated, since there are subclinical cases. Even with factors strongly associated with its onset, such as systemic conditions and emotional stress, about one-third of the cases remain without clear etiology. ${ }^{9}$

The nutritional impact has received more attention in the last years, since the cells of the matrix of the hair follicle are among those of faster division, and therefore, more sensitive to any nutritional reduction, where iron deficiency is the most proven, both by its frequency and by its importance, being a cofactor of DNA synthesis. ${ }^{10}$

Table 3 Hair density represented by number of counted hairs in standard area

\begin{tabular}{|c|c|c|c|c|c|c|}
\hline & \multicolumn{2}{|c|}{ Initial assessment } & \multicolumn{2}{|l|}{$\underline{D 90}$} & \multicolumn{2}{|l|}{ D I 80} \\
\hline & I & II & I & II & $\mathbf{I}$ & II \\
\hline Mean & $235.4 I \pm 59.99$ & $242.88 \pm 71.46$ & $232.88 \pm 66.04(0.747 \%)$ & $251.89 \pm 86.03$ (3.71\%) & $263.44 \pm 89.62(1 \mathrm{l} .09 \%)^{\mathrm{a}}$ & $261.73 \pm 89.67(7.76 \%)$ \\
\hline
\end{tabular}

Notes: aLevel of significance of $5 \%$. Hypothesis: non-inferiority among treatments.

Abbreviations: I, group treated with Eximia Fortalize Kera D ${ }^{\circledR}$; II, group treated with comparator; D90, 90-day evaluation; DI80, I80-day evaluation.

Table 4 Comparison by Mann-Whitney $U$ test between groups I and 2 in D90 in relation to D0 ( $n=92)$

\begin{tabular}{|c|c|c|c|}
\hline Subjective parameters & I (\%) & II (\%) & P-value \\
\hline Hair loss improvement & 70.22 & 77.78 & 0.109 \\
\hline Hair shine & 59.58 & 53.33 & 0.330 \\
\hline Hair strength & 78.72 & 57.78 & $0.022^{\mathrm{a}}$ \\
\hline Convenience of use in terms of the amount of tablets/capsules ingested per day & 91.48 & 74.34 & $0.012^{\mathrm{a}}$ \\
\hline Ease of swallowing of tablets/capsules & 82.98 & 86.67 & 0.706 \\
\hline Absence of residual taste from tablets/capsules & 72.34 & 40.00 & $0.00 \mathrm{I}^{\mathrm{a}}$ \\
\hline
\end{tabular}

Notes: aLevel of significance of $5 \%$. Hypothesis: non-inferiority among treatments.

Abbreviations: I, group treated with Eximia Fortalize Kera $\mathrm{D}^{\circledR}$; II, group treated with comparator; D90, 90-day evaluation; D0, initial time.

Table 5 Comparison by Mann-Whitney $U$ test between groups I and 2 at DI80 in relation to D0 ( $n=92)$

\begin{tabular}{|c|c|c|c|}
\hline Subjective parameters & I (\%) & II (\%) & P-value \\
\hline Hair loss improvement & 82.98 & 80.00 & 0.866 \\
\hline Hair shine & 76.60 & 77.78 & 0.940 \\
\hline Hair strength & 80.85 & 84.44 & 0.346 \\
\hline Convenience of use in terms of the amount of tablets/capsules ingested per day & 93.62 & 67.78 & $0.006^{\mathrm{a}}$ \\
\hline Ease of swallowing of tablets/capsules & 85.11 & 88.89 & 0.829 \\
\hline Absence of residual taste from tablets/capsules & 74.47 & 66.22 & 0.214 \\
\hline
\end{tabular}

Notes: ${ }^{a}$ Level of significance of $5 \%$. Hypothesis: non-inferiority among treatments.

Abbreviations: I, group treated with Eximia Fortalize Kera ${ }^{\circledR}$; II, group treated with comparator; D0, initial time; DI80, I80-day evaluation. 
$\mathrm{V}$ Diet plays an important role in TE. Very restrictive diets with low protein content may lead to reduced matrix synthesis and precipitate telogen-phase hair loss. ${ }^{11}$

The results of the present study demonstrate that nutritional supplementation may be beneficial in TE when there are no associated hormones, even in monotherapy. Both treatments showed positive significant results in the parameters evaluated in the study.

Some publications have demonstrated that even in normal levels, micronutrients such as zinc or iron, can be lower in female pattern hair loss, male pattern hair loss, or TE. ${ }^{12,13}$ Probably, reduced levels of other micronutrients play a key role in TE even though in normal levels.

However, the product evaluated in group 1, Eximia Fortalize Kera $\mathrm{D}^{\circledR}$, in addition to being similar to the comparator, even offering an association of nutrients in physiological concentrations, demonstrated superior efficacy over time compared to group 1 , which used the comparator.

In the clinical evaluation $(P<0.05)$, for the clinical parameters of hair quality, hair shine, and hair strength, the latter was also perceived earlier by the study participant, in as early as 90 days of use.

In the trichoscopic evaluation, a higher percentage increase of hair density was observed over time, being statistically significant $(P<0.05)$.

In the patient subjective evaluation, better dosage convenience is considered important to the adherence of the patient to therapy, especially for a longer time.

Also regarding tolerance, the group treated with the comparator product had dyspeptic symptoms with higher frequency; although there is no statistical significance, three patients had symptoms of gastrointestinal discomfort. This adverse reaction profile is also described for this product in the product package insert and in the literature. ${ }^{14}$

The presence of para-aminobenzoic acid (PABA) may possibly be related to these findings, besides the size of the tablet and the dosage profile. Oral PABA is related to dyspeptic reactions in addition to photosensitization. ${ }^{15,16}$

The association of nutrients in physiological concentrations therefore seems to not only equate to associations with higher concentrations, but can also effectively show superior results, since it contains ingredients whose participation in keratinogenesis and pillar cycle are essential.

Even borderline conditions of anemia can have repercussions on TE precipitation, and universal research has not yet found a recommendation with sufficient evidence; clinical context (menstrual losses, vegetarian practices, etc) should also be taken into account. ${ }^{17}$
Zinc is involved in the synthesis of proteins and nucleic acids, having an important role in various metabolic pathways and cellular functions. Specifically in the pilous follicle, zinc is a potent inhibitor of pilous follicle regression in an animal model. ${ }^{18}$

B-complex vitamins, in particular biotin and folic acid, play a decisive role in the hair cycle. Borderline deficiencies may be present in patients under restrictive diets for weight loss, also interfering with the differentiation of epidermal cells; however, the B-complex vitamins act in synergy, and the conditions of point deficiency of one of them alone are rare. ${ }^{19}$

The possible benefit of the use of antioxidants in TE is supported by the associated etiological factors, especially when there are associated systemic inflammatory processes.

Nutrients with this function should be carefully administered, preferably at physiological doses, in order to preserve redox balance. ${ }^{20}$

Similarly, the role of vitamin D has been much discussed in several pathologies, including TE; a study involving 80 women with TE demonstrated significantly lower levels in relation to the control group, although the mechanism of action has not yet been established. ${ }^{21}$

In TE, the lack of amino acids is also frequent, many of them being constituents of keratin; a study of 100 patients with alopecia demonstrated that histidine deficiency was present in $77.78 \%$ of participants with TE, while leucine deficiency, a branched-chain amino acid, appeared in $98.15 \%$ of TE carriers. Cysteine deficiency, the main constituent amino acid of keratin, was present in half of the participants with TE, and there was no symptomatology of the deficiency except hair loss itself, in addition to a history suggestive of low ingestion. ${ }^{22}$

Considering that the constituent amino acids of keratin are crucial in hair synthesis and may lead to TE, the formulation studied here contains both essential and nonessential amino acids that make up keratin, including cystine, the main component of keratin, formed from cysteine.

This study demonstrated that in idiopathic TE, the nutritional component should be suspected; and the supplementation of an association of nutrients in recommended daily intake can lead to a significant improvement of the condition from the first trimester of use. The use of an association with proven efficacy and a safety profile and posologic convenience facilitate its indication and patient adherence.

\section{Disclosure}

Ludmila Coelho Donato and Camila Sirieiro Abreu Melo are employees in Farmoquímica S.A. Flávia Alvim Sant'Anna Addor reports no conflicts of interest in this work. 


\section{References}

1. Gilmore S, Sinclair R. Chronic telogen effluvium is due to a reduction in the variance of anagen duration. Australas J Dermatol. 2010;51(3):163-167.

2. Whiting DA. Chronic telogen effluvium: increased scalp hair shedding in middle-aged women. J Am Acad Dermatol. 1996;35(6):899-906.

3. Grover C, Khurana A. Telogen effluvium. Indian J Dermatol Venereol Leprol. 2013;79(5):591-603.

4. Rushton DH. Nutritional factors and hair loss. Clin Exp Dermatol. 2002;27(5):396-404.

5. Addor FAS, Bombarda PCP, Bombarda Júnior MS, Abreu FF. Influência da suplementação nutricional no tratamento do eflúvio telógeno: avaliação clínica e por fototricograma digital em 60 pacientes [Influence of nutritional supplementation in the treatment of telogen effluvium: clinical and digital phototricogram evaluation in 60 patients]. Surg Cosmet Dermatol. 2014;6(2):1316. Portuguese.

6. Kil MS, Kim CW, Kim SS. Analysis of serum zinc and copper concentrations in hair loss. Ann Dermatol. 2013;25(4):405-409.

7. Cheung EJ, Sink JR, English III JC. Vitamin and mineral deficiencies in patients with telogen effluvium: a retrospective cross-sectional study. J Drugs Dermatol. 2016;15(10):1235-1237.

8. Petri H, Pierchalla P, Tronnier H. Die Wirksamkeit der medikamentösen Therapie bei strukturellen Läsionen der Haare und bei diffuser Effluvium - vergleichende Doppelblindstudie. [The efficacy of drug therapy in structural lesions of the hair and in diffuse effluvium - comparative double blind study]. Schweiz Rundsch Med Prax. 1990;79(47):1457-1462. German.

9. Trüeb RM. Effect of ultraviolet radiation, smoking and nutrition on hair. Curr Probl Dermatol. 2015;47:107-120.

10. Piccardi N, Manissier P. Nutrition and nutritional supplementation: impact on skin health and beauty. Dermatoendocrinol. 2009;1(5):271-274.
11. Goette Dk, Odom RB. alopecia in crash dieters. JAMA. 1976;235: 2622-2623.

12. Addor FAS. Nutrient supplementation influence on keratinocytes metabolism: an in vitro study. Surg Cosmet Dermatol. 2012;4(2): $150-154$.

13. Addor FAS, Bombarda PCP, Bombarda Júnior MS, Abreu F. Influence of nutritional supplementation in the treatment of telogen effluvium: clinical assessment and digital phototrichogram in 60 patients. Surg Cosmet Dermatol. 2014;6(2):1316.

14. Levine RA. Steatorrhea Induced by Para-aminosalicylic Acid. Ann Intern Med. 1968;68:1265-1270.

15. Kobler E, Bühler H, Nüesch HJ, Deyhle P. [Drug-induced oesophageal ulcers]. Dtsch Med Wochenschr. 1978 23;103(25):1035-1037.

16. Stoevesandt J, Kürzinger N, Bröcker EB, Trautmann A. Uro-dermatological problems of a construction worker: paraaminobenzoic acid as a systemic photosensitizer. Eur J Dermatol. 2010;20(2):217-219.

17. Trost LB, Bergfeld WF, Calogeras E. The diagnosis and treatment of iron deficiency and its potential relationship to hair loss. $\mathrm{J} \mathrm{Am} \mathrm{Acad}$ Dermatol. 2006;54(5):824-844.

18. Plonka PM, Handjiski B, Popik M, Michalczyk D, Paus R. Zinc as an ambivalent but potent modulator of murine hair growth in vivo - preliminary observations. Exp Dermatol. 2005;14(11):844-853.

19. Trüeb RM. Serum biotin levels in women complaining of hair loss. Int J Trichology. 2016;8(2):73-77.

20. Addor FAS. Antioxidants in dermatology. An Bras Dermatol. 2017; 92(3):356-362.

21. Gowda D, Premalatha V, Imtiyaz DB. Prevalence of nutritional deficiencies in hair loss among Indian participants: results of a cross-sectional study. Int J Trichology. 2017;9(3):101-104.

22. Ramos PM, Miot HA. Female pattern hair loss: a clinical and pathophysiological review. An Bras Dermatol. 2015;90(4):529-543.
Clinical, Cosmetic and Investigational Dermatology

\section{Publish your work in this journal}

Clinical, Cosmetic and Investigational Dermatology is an international, peer-reviewed, open access, online journal that focuses on the latest clinical and experimental research in all aspects of skin disease and cosmetic interventions. This journal is included on PubMed. The manuscript management system is completely online

\section{Dovepress}

and includes a very quick and fair peer-review system, which is all easy to use. Visit http://www.dovepress.com/testimonials.php to read real quotes from published authors 